\title{
The nature of nano-confined geofluids: Defining the dielectric constant via molecular dynamics simulations
}

\author{
ALIREZA CHOGANI AND OLIVER PLÜMPER
}

Utrecht University

Presenting Author: a.chogani@uu.nl

Fluid-driven metamorphism is often associated with the formation of nanopores, most of which are less than $100 \mathrm{~nm}$ in size [1.2]. Critical physicochemical properties of the nanoconfined fluid, such as the dielectric constant $\varepsilon$, may differ significantly when compared to its corresponding bulk fluid [3]. Hence, there is a need for a comprehensive assessment of the dielectric constant of nanoconfined fluids in geological systems. There are various parameters including rock material, pressure, temperature, size and shape of the nanopores, concentration and type of solvents that may influence the dielectric behavior of polar fluids. These local effects can have a profound impact on mineral solubility and fluid transport properties. Here we use molecular dynamics (MD) simulations to investigate dielectric behavior of water confined between two brucite layers. Two rigid models $\mathrm{SPC} / \mathrm{E}$ and $\mathrm{SPC} / \varepsilon$ are employed where the latter is developed specifically to predict the dielectric constant of water. The static dielectric constant is calculated as a function of pressure and temperature of the Earth's crust and estimated using a relation based on fluctuations of the total dipole moment. In all cases, a rise in the static dielectric constant is seen through the decreasing temperature or increasing pressure. To investigate the dielectric constant tensorial behavior, its component is calculated in the direction perpendicular to the brucite layers. With the reduction of the width of the nanochannel, i.e., smaller nanopores, the perpendicular component of dielectric constant declines. We use these MD-based estimates of the dielectric constant to assess its impact on mineral solubility changes in nanoporous metamorphic rocks.

References

[1] Tutolo et al. (2016). Geology, 44(2), 103-106.

[2] Plümper et al. (2017). Nature Geoscience, 10(9), 685-690.

[3] Motevaselian et al. (2020). ACS Nano, 14(10), 12761-12770. 